\title{
New Methods for Helicopter for Free Flight Inside Clouds and Precipitation Enhancement
}

\author{
Richard O. Claus ${ }^{1}$, Tamara V. Tulaikova ${ }^{2}$ \\ ${ }^{1}$ NanoSonic Inc. Pembroke, Virginia, USA \\ ${ }^{2}$ Wave Research Center at Prokhorov General Physics Institute, Moscow, Russia
}

Email address:

tulaikova@gmail.com (T. V. Tulaikova)

To cite this article:

Richard O. Claus, Tamara V. Tulaikova. New Methods for Helicopter for Free Flight Inside Clouds and Precipitation Enhancement. American Journal of Environmental Protection. Special Issue: New Technologies and Geoengineering Approaches for Climate. Vol. 5, No. 3-1, 2016, pp. 1-9. doi: 10.11648/j.ajep.s.2016050301.11

\begin{abstract}
The method of considerable decrease the $\mathrm{CO}_{2}$ concentration in atmosphere based on precipitation enchainment is considered. Nowadays approaches for cloud reorganization are very important, because the sum of $\mathrm{CO}_{2}$ and water vapor that collected in clouds makes $95 \%$ mass of greenhouse gases. Usage of helicopter for free flight inside natural clouds is considered in comparison with traditional airplanes. The method for anti-icing coating for helicopter and laboratory experiments with formulas for the cloud droplets adhesion/separation are presented. New technology for fast drying of air in medium near helicopter rotor is outlined.
\end{abstract}

Keywords: Acoustics, Helicopter, $\mathrm{CO}_{2}$, Clouds, Precipitation Enhancement

\section{Introduction and Background}

Development of new technical methods for free atmosphere purification from greenhouse gases is very important in modern world and mitigation approach is necessary in order to combat the imminent dangers related to climate changes. Our climate is changing; this fact was proved by IPCC reports based on detailed world's science [1] The global warming was caused by exceed of greenhouse gases, and annual $\mathrm{CO}_{2}$ emission producing by world industry provide evident concentration increase in atmosphere at each year. The sum of $\mathrm{CO}_{2}$ and water vapor that collected in clouds makes $95 \%$ mass of greenhouse gases nowadays. Currently the role of clouds becomes important, so the methods of cloud reorganization are needed. Traditionally, humans tried use the natural clouds to produce a lot of water for agriculture at the necessary moments. The development of these methods are based on the fact that a typical cloud can contain thousands tons of water. Modern methods for precipitation enhancements have been developed in different countries around the world with wide practice [2]. The most popular current technologies for precipitation enhancement are based on airplanes those are sprinkling a chemical reagents into cloud. Note that a helicopter can serve to this purpose much better, because it is less speedy and more maneuverable in comparison with an airplane.

Our approach incorporates the possibility of stepwise $\mathrm{CO}_{2}$ purification by cloud modification [3]. The first stage involves spraying of alkaline compounds, such as $\mathrm{KOH}$ or etc. inside cloud via an airplane or helicopter. The alkaline reagents significantly increase the solubility of $\mathrm{CO}_{2}$ in water droplets due to increase the $p H$ in cloud droplets, and rain droplets become saturated by atmospheric $\mathrm{CO}_{2}$ during their gravitational fall from cloud to the ground. The $\mathrm{CO}_{2}$ solubility significantly grows due to increase of $\mathrm{pH}$, therefore ions concentrations of $\left[\mathrm{HCO}_{3}{ }^{-}\right]$and $\left[\mathrm{CO}_{3}{ }^{2-}\right]$ increase in 10 and 100 times accordingly by each unit of $p H$. This method can be conducted in areas of the free atmosphere step by step, so its application on the global scale was analyzed for the whole Earth. The future technology can compensate for annual carbon emission by method application at $0.4 \%-$ $0.08 \%$ of our planet surface then $p H=10-11$, [4]. The advantage of proposed approach is a good response in plant growth to the precipitation from modified clouds; one can see the strong vegetation growth by enriched watering at Fig. 1 here and in [5]. To emulate the process of $\mathrm{CO}_{2}$ absorption in water droplets during an indoor experiment, the similar changes were made in alkaline solution during long-time diffusion according to formula $L_{D} \approx(D \cdot t)^{1 / 2}$, there a diffusion coefficient in water is $D$, and $L$ is a water layer depth. The 
$\mathrm{KOH}$ was dissolved in water and resulting mixture was kept indoors during several hours $t=L_{D}^{2} / D$, as a result the solution has carbon ions of $\mathrm{HCO}_{3}{ }^{-}, \mathrm{CO}_{3}{ }^{2-}$ and $\mathrm{K}+$. The initial $p H=12$ was kept in these experiments. After saturation time $t$, the plants in brown pot were given the resulting solution and pure water was added to control blue pot (right side) with the same plants. These two watering processes were repeated regularly every day during one month. The experimental plants are beetroots (10 grains), carrots (20 grains), and parsley (2 grams) in each of plant pots. One can see a strong vegetation growth by enriched watering according to proposed method for all studied plants, Fig.1. Nowadays precipitation in industrial regions have led to soil deterioration up to $\mathrm{pH}=4$ or less.

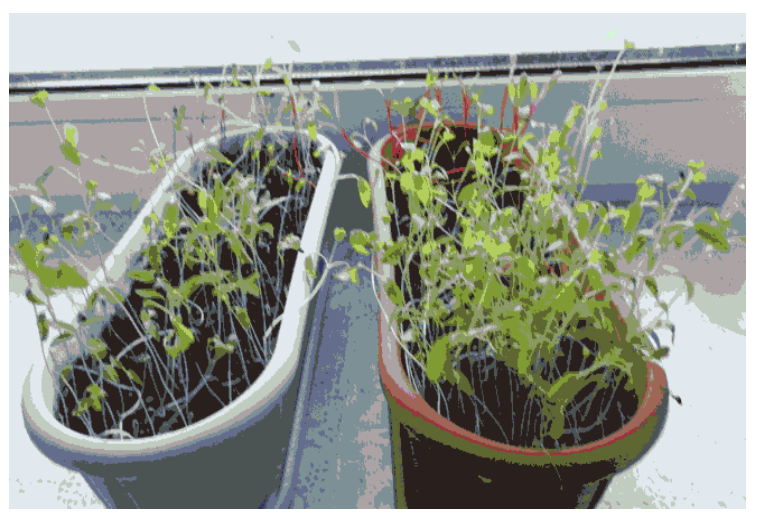

Figure 1. The indoor plants after 30 days correspondingly; plants in a right (brown) flower pot were watered by solution with carbon ions $\mathrm{CO}_{3}{ }^{2-}$, $\mathrm{HCO}_{3}{ }^{-}$and $\mathrm{K}^{+}$

Other advantages of the method are based on a substantial joint use of surface of all small droplets in the current precipitation volume. The joint set of water droplets increases the available surface for gas/liquid interactions for fast and complete $\mathrm{CO}_{2}$ absorption in the air volume under cloud. The process can be simulated in advance to take out from the atmospheric volume the desirable mass of carbon.

The idea of regional weather modification by precipitation enhancement was described earlier [6]. Nowadays the most popular methods are the sprinkling of hygroscopic particles or special solutions for warm clouds [7] and the introduction of glaciogenic substances into cold clouds [8] from an airplane. In addition to it, special acoustic devices could be used to accelerate the coalescence of water droplets in clouds. The background is the fact that acoustics provides high mobility for droplets, because they become involved in air vibrations inside sound waves with the best coalescence as a result. Previous acoustical experiments [9] and further analysis were carried out to show efficiency for acoustics inside clouds directly in the region with oversaturated water steam by using a modern airplanes or helicopter. The most effective is a joint utilization of two methods at the same time, which means that hygroscopic particles and acoustic influence would be directly applied inside one cloud area. This result depends on different areas of both considered methods. Dynamics of different hygroscopic particles show that only first 15 - 20 seconds demonstrate fast changes in the main cloud characteristics such as super saturation and spectrum dispersion. This is accompanied by an increase of the radii inside small drops from $0.01-0.1 \mu \mathrm{m}$ up to $1-3 \mu \mathrm{m}$. So after the first major variations these parameters increase very slowly, hence significant changes in spectrum of cloud and precipitation increase up to 50 minutes [7]. Later the droplets should be triggered by sound waves in the form of vibrations to provide wide condensation and coalescence up to a drop size of $100 \mu \mathrm{m}$, and after this point gravity can predominate. Acoustic method was analyzed to obtain fast droplets coalescence inside nature clouds up to precipitation with big droplet sizes and gravity sedimentation [10]. Optimal acoustic generator is proposed; also optimal regimes of acoustical powers for typical cloud droplets are calculated in [10] and presented here at Fig.2. The Figure 2 demonstrates examples of the three acoustical ranges, as follows: (1) $f=20 \mathrm{~Hz}$ with lowest power $Q=175 \mathrm{~W} / \mathrm{m}^{2}$; (2) $f$ $=50 \mathrm{~Hz}$ with $Q=800 \mathrm{~W} / \mathrm{m}^{2}$ and (3) $f=100 \mathrm{~Hz}$ with $Q=$ $2500 \mathrm{~W} / \mathrm{m}^{2}$. We investigated the low frequency range from $f$ $=50-100 \mathrm{~Hz}$ in order to provide droplet amplitude in a range $L \sim 0.5-2.5 \mathrm{~mm}$ for coalescence possibility for typical clouds. Additional analysis was performed and introduced the appropriate altitude changes relative to the main physical parameters in the model, as follows: air density $\left(\rho_{\mathrm{a}}\right)$, viscosity $(\eta)$, sound velocity $\left(C_{a}\right)$, and air temperature depending on $\mathrm{km}$-altitudes, and an improvement decrease in necessary acoustic power was observed. Usage of sound source at the altitude of $2-6 \mathrm{~km}$ within the cloud by airplane or helicopter provides the high droplet coalescence to get precipitation.

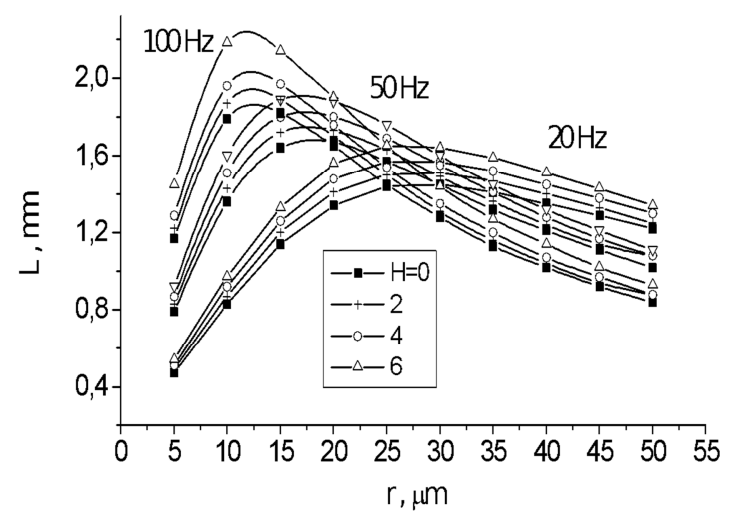

Figure 2. Amplitudes L for vibrated droplets in acoustic field; droplet's radii are $r=5-50 \mu \mathrm{m}$ and clouds are located at altitudes $H=0,2,4$, or $6 \mathrm{~km}$. Acoustic regimes are: $f=100 \mathrm{~Hz}$ and $Q=2.5 \mathrm{~kW} / \mathrm{m}^{2} ; f=50 \mathrm{~Hz}$ and $Q=800$ $\mathrm{W} / \mathrm{m}^{2} ; f=20 \mathrm{~Hz}$ and $Q=175 \mathrm{~W} / \mathrm{m}^{2}$.

The additional acoustic action can be effectively performed done by helicopter. The main advantage is the following. Rotor blade of the helicopter rotates with maximal angular velocity, so the linear velocity at the end of each blade reaches the speed of sound. Typical helicopters blade has a length of 10 meters and its partial speed varies linearly from 0 to $300 \mathrm{~m} / \mathrm{sec}$ for further estimation here. Note that helicopter is moving forward with some speed, for example $100 \mathrm{~m} / \mathrm{sec}$; then supersonic motion of one blade is produced 
in small time periods. This effect takes place because there are the same directions of helicopter motion and one of its blades moving in the same direction with its own speed. It means that each of the blades overcomes the supersonic regimes and produces the shock wave. The shock wave has a steep front and bigger difference of pressure and local temperature comparable to surroundings. A shock way is a very best acoustical source for droplets in a cloud, such wave pushes the droplets to effective coalesce. Fast condensation inside the shock wave can be seen many times directly and also in photographs when airplane breaks the sound barrier. The helicopter has multiple advantages in comparison with airplane, and its icing immunity can be used successfully for meteorological applications, in particular for precipitation advantages. Techniques for anti-icing usage and improvement will be considered here in parts 3 and 4 .

The modified precipitation droplets provide effective transport of $\mathrm{CO}_{2}$ from the under-cloud atmospheric volumes to the ground and further more to soil, ground water and plants. Therefore, new systems for cloud management and regularly precipitation enhancement are necessary for recovery the atmosphere, soil, ocean and climate as a result.

\section{The Precipitation Method for Carbon Transport from Atmosphere}

The effect strongly relates to artificial changes of $p H$ level in cloud water content. In nature also the ocean waters keeps significant masses of dissolved $\mathrm{CO}_{2}$ due to its alkaline $\mathrm{pH}$-level that is about $p H=8.3$. There are known description for the insoluble and dissociated portions of the weak acid that remains after attaining the equilibrium of saturation for water by $\mathrm{CO}_{2}$ as follows:

$$
\begin{gathered}
\mathrm{CO}_{2}+\mathrm{H}_{2} \mathrm{O} \Leftrightarrow \mathrm{H}_{2} \mathrm{CO}_{3}, \mathrm{HCO}_{3}^{-} \Leftrightarrow \mathrm{CO}_{3}^{2-}+\mathrm{H}^{+} \\
\mathrm{H}_{2} \mathrm{CO}_{3} \Leftrightarrow \mathrm{HCO}_{3}^{-}+\mathrm{H}^{+}
\end{gathered}
$$

The natural rain can't provide the carbon transport from atmosphere to the ground. In typical natural precipitation at the level $p H \approx 5.6$ the concentrations of the dissolved and dissociated portions are titled as follows: $\left[\mathrm{H}_{2} \mathrm{CO}_{3}\right]$, $\left[\mathrm{HCO}_{3}^{-}\right]$, $\left[\mathrm{CO}_{3}{ }^{2-}\right]$, that are very small in this case. The gas solubility strongly increases with higher levels of $p H$. The effect in a cloud with $3 \mathrm{mkg} / \mathrm{m}^{3}$ of $\mathrm{NH}_{3}$ was shown by Yunge [11] and Rasool [12], so the medium has neutral $p H=7$ but with appropriate increase for the concentration of dissolved ions. The partial concentrations are $\left[\mathrm{H}_{2} \mathrm{CO}_{3}\right]=0.71 \mathrm{mg} / \mathrm{l} ;\left[\mathrm{HCO}_{3}{ }^{-}\right]$ $=3.3 \mathrm{mg} / \mathrm{l},\left[\mathrm{CO}_{3}{ }^{2-}\right]=10^{-3} \mathrm{mg} / \mathrm{l}$. The ratios of carbon in the first, second and third of the listed compounds are 0.1935; $0.1967 ; 0.20$, correspondingly, that should be multiplied to concentration to find partial carbon masses and total one. The masses of carbon in 1 liter of water then $p H=7$ are as follows: $0.1373 \mathrm{mg} / l, 0.6491 \mathrm{mg} / L, 2 \cdot 10^{-4} \mathrm{mg} / l$, and their sum is $\mathrm{C}_{\mathrm{pH} 7}=0.7866 \mathrm{mg} / \mathrm{l}$. The ionic concentrations of $\left[\mathrm{HCO}_{3}{ }^{-}\right]$ and $\left[\mathrm{CO}_{3}{ }^{2-}\right]$ increase in 10 and 100 times accordingly by each unit of $p H$. We performed similar calculations assuming that we increase $p H$ in atmospheric water droplets up to 10,11 , etc [3-5]. The total mass of carbon in 1 liter of water with $p H$ $=10$ is $C_{p H 10}=849.1 \mathrm{mg} / l$, see row 2 in Table 1 .The carbon compound culd be measured in water using one of standard methods, see [14] or ets.

Let's assume the initial cloud modification to $p H=10$, and precipitation layer $h_{w}=10 \mathrm{~mm}$ at surface $S=1 \mathrm{~km}^{2}$, then it gives $10^{7}$ liters. The total removed carbon mass will be $M=$ $C_{p H ! 0} h_{w} \mathrm{~S}=8.5$ tons in such modified precipitation water during one rain. Then $p H=11$ the same rain will transport the 265 tons of carbon ions that is equal to 972 tons of $\mathrm{CO}_{2}$ to the ground during this one rain.

Now, let's analyze the example volume of purified air in the atmosphere $U=1 \mathrm{~km}^{3}$ under rain, where $h=1 \mathrm{~km}$ is the altitude of the cloud over land and the unit square of the surface is $1 \mathrm{~km}^{2}$. The reduced $\mathrm{CO}_{2}$ concentration was estimated by dissolving the carbon mass in alkaline rain using an assumption that the initial concentration of $\mathrm{CO}_{2}$ was even under the cloud. The molar mass of $\mathrm{CO}_{2}$ is 44 , so the share of carbon in it is 0.2727 . Using previous calculations, the atmospheric concentrations could be reduced to the following values: $C_{a=}=\left(C_{p H 10} / 0.2727\right) \cdot\left(h_{w} / h\right)$. Similar calculations with different $p H$-level, water layer $h_{w}$, and cloud altitude, $h$, show different possible warrants for utilization. For example, the concentration of removed $\mathrm{CO}_{2}$ in this air volume should be approximately $420 \mathrm{ppm}$ if the $\mathrm{pH}=10.3$ in rain water then $h_{w} /$ $h=10^{-4}$. If the height of the clouds over the land was greater than $1 \mathrm{~km}$, for example $h=6 \mathrm{~km}$, then all of the data of concentrations should be divided by 6 due to the increase in purified air volume. For this case and for a $p H=10.8$, atmospheric purification will be complete again with removing concentration $C_{a_{-}}=403 \mathrm{ppm}$, and the same precipitation layer is $h_{w}=10^{-1} \mathrm{~m}$. It is suggested the uniform $\mathrm{CO} 2$ vertical distributions, of cause the local effect is persists up to the moment of new injections or winds.

Key advantages for proposed method should be eliminated for alkaline rains in comparison with ocean water. First, due to small dimensions of precipitation droplets the falling time exceed the saturation time for the gas molecules inside precipitation droplets. The largest radius has been estimated as $3 \mathrm{~mm}$ up to drop disintegration. However, at the beginning of the precipitation the droplets have grown up to a radius of more then $r>100 \mu \mathrm{m}$ the stated values for gravitational sedimentation. The fall velocity $V_{g}(r)$ can be approximated roughly using the stationary speed of droplet gravitation sedimentation as follows: $V_{g}(r)=\sqrt{2 r g r_{w} / r_{a}}$, where $\rho_{\mathrm{a}}$ and $\rho_{\mathrm{w}}$ are the density for air and water respectively, and $g$ is the acceleration due to gravity. The falling time for droplet can be estimated as $t_{h} \approx h / V_{g}(r)$ at the initial minimal altitude of $h=1$ $\mathrm{km}$. During $\mathrm{CO}_{2}$ solubility, the next step demands estimations for the saturation time $t_{a w}$ in falling droplets. The process of a concentration establishment described by the law: $t_{a w} \approx\left(4 \pi r^{3} / 3\right) /\left(K_{a w} 4 \pi r^{2}\right)=r /\left(3 K_{a w}\right)$, the time depends on the ration of the aerated volume $U_{l}$ for a drop to the area of its surface $S_{I}$ in a gas flow [13]. The process also depends on the 
gas exchange constant $K_{a w}$ at the gas-liquid interface, the constant $K_{a w}(V)$ depends on the flow speed $V=V_{g}(r)$. In the past, the intensity of $\mathrm{CO}_{2}$ gas exchange has been measured depending on the velocity of air flow $V$ over the water surface [15-17] that gives $K_{a w}(5) \approx 6.4 \cdot 10^{-3} \mathrm{~cm} / \mathrm{ssthen} V=5 \mathrm{~m} / \mathrm{s}$, and etc. Unifying the previous equation with measured constraint yields the saturation time $t_{a w}=6.3 \mathrm{sec}$, but falling time is $t_{h l}=$ $257 \mathrm{sec}$ for droplet radius $r=1 \mathrm{~mm}$, and the similar for other sizes of droplets. Since $t_{a w}<<t_{h}$ there is enough time for the saturation of typical rain droplets by $\mathrm{CO}_{2}$ molecules during their flight to the ground from a $1 \mathrm{~km}$ altitude.

Analyses and calculations demonstrate the big total number of droplets in unit volume and their big summarized surface to contact with air during droplets flight. The analysis is based on the Marshall-Palmer approximation for the droplets spectrum with radii interval $r_{m}=0.05-3 \mathrm{~mm}$ during precipitation [3]. The volume of received water $U_{I}$ was calculated as a sum of all of the falling droplets of the water layer $I=3 \mathrm{~mm}$ on a ground surface $A=1 \mathrm{~m}^{2}$ during $T=1$ hour. The calculations provide the total number of falling droplets in mentioned water unit, for a rain this number is $N_{r}=4.02 \cdot 10^{11}$, but for a drizzle $N_{d} \approx 6.76 \cdot 10^{11}$ inside 3 liters of precipitation water. These results prove that a set of rain droplets have a large surface for gas/liquid interactions as compared to the ocean's plane surface. Then, each falling droplet runs at an air cylinder with a minimal volume length $h$ and a ground area $\pi r_{m}{ }^{2}$. The cylinder surface $S_{m} \approx h 2 \pi r_{m}$ means that air/water interface increases the contact for rainwater and purified air. The total sum of this surface for all droplets in considered water unit is $S_{r}=2 \cdot 10^{8} \mathrm{~m}^{2}$ for rain, but the same units for a box of air volume has $4 \cdot 10^{3} \mathrm{~m}^{2}$ with a vertical altitude of $h=1 \mathrm{~km}$ and a background $A=1 \mathrm{~m}^{2}$. Important note is that due to the small concentration of $\mathrm{CO}_{2}$ molecules $(400 \mathrm{ppm})$, the probability of their collision with small water drops is low within clouds due to their small sizes. Therefore, a strong decrease in the concentration of $\mathrm{CO}_{2}$ within clouds will not be achieved at the first step, and the alkaline reserve of a reagent introduced into the clouds will not be spent. The erosion of $\mathrm{CO}_{2}$ as well as a significant reduction in air concentration will certainly occur in the sub-cloud volume via precipitation.

The $\mathrm{KOH}$ substance has hygroscopic properties such as that it absorb water around aerosol droplets due to condensation in the supersaturated media of clouds. The initial $\mathrm{KOH}$ aerosol droplets contain an alkaline saturated solution with a concentration $n_{0}$. Further analyses and calculations were performed in order to provide the average $p H$-levels in cloud water. The $\mathrm{KOH}$ reacts in water as follows:

$$
\begin{gathered}
\mathrm{KOH} \rightarrow \mathrm{K}^{+}+\mathrm{OH}^{-} \\
\mathrm{H}^{+}+\mathrm{OH}^{-} \Leftrightarrow \mathrm{H}_{2} \mathrm{O}, K_{w}=\left[\mathrm{H}^{+}\right] \cdot\left[O \mathrm{H}^{-}\right]
\end{gathered}
$$

Formulas for the quantity mass of $\mathrm{KOH}$ are the following:

$$
n_{m o l}=\left[O H^{-}\right]
$$

The choice of a $\mathrm{pH}=10$, for example, demands that $\left[\mathrm{OH}^{-}\right]$ $=10^{-4}$, and the molar concentration now becomes $10^{-4} \mathrm{~mol} / \mathrm{L}$ according to last equation in (2). The mass concentration yields the value $n_{\mathrm{KOH}}{ }^{\left({ }^{\mathrm{HH} 10)}\right.}=56 \cdot 10^{-4} \mathrm{~g} / L$, by taking into account the $\mathrm{KOH}$ molar mass of 56 . The data can be used in the following manner: if the liquid water content in the medium of a unit cloud is $W=1 \mathrm{~g} / \mathrm{m}^{3}$, whereas $1 \mathrm{~m}^{3}$ of an air cloud contains $1 \mathrm{~cm}^{3}$ of pure water, and $5.6 \mathrm{mkg}$ of masses should be introduced into a $1 \mathrm{~m}^{3}$ of air within the cloud in order to ensure the appropriate $p H=10$. The cloud volume with $1 \mathrm{~km}^{3}$ of volume and $W=1 \mathrm{~g} / \mathrm{m}^{3}$ need $5.6 \mathrm{~kg}$ to get $p H$ $=10$ in a volume about $1 \mathrm{~km}^{3}$ of cloud. The $56 \mathrm{~kg}$ provides $p H=11$ in the same cloud. For more accuracy the aerosol dynamics in cloud media was calculated in [3].

The proposed method can be applied for the whole Earth on the global scale. The surface area of our planet is $A_{E}=$ $5,1 \cdot 10^{8} \mathrm{~km}^{2}$ and the average annual layer of precipitation is $h_{w}=1,000 \mathrm{~mm}$. Using algorithm and equations in [3-5] one can estimate the mass of removed atmospheric carbon $\left(m_{C}{ }^{l}\right)$ in 1 meter of precipitation water measured per surface $1 \mathrm{~m}^{2}$, these are presented at $2^{\text {nd }}$ row of Table 1 , and $p H=10-11$ is indicated at $1^{\text {st }}$ row. The estimated masses of $\mathrm{CO}_{2}$ in $1 \mathrm{~m}^{3}$ of water are presented at $3^{\text {th }}$ row, so the carbon ratio in $\mathrm{CO}_{2}$ is 0.2727 . The calculated mass of formed carbon at the surface $A_{E}$ of the whole planet is $M_{C}^{A}=m_{C}{ }^{l} \cdot A_{E}=4.3 \cdot 10^{14} \mathrm{~kg}$ then $p H$ $=10$, as listed at the $4^{\text {th }}$ row of the Table 1 . Hence the amount of carbon oxide is $M_{C 02}^{A}=M_{C}^{A} / 0.2727$ and its value listed at the $5^{\text {th }}$ row of the Table. In 2010 the global $\mathrm{CO}_{2}$ emission reached an amount of $3.06 \cdot 10^{13} \mathrm{~kg}$. For further calculations let's assume the global annual emission of $\mathrm{CO}_{2}$ as the $\mathrm{AE}=$ $3.2 \cdot 10^{13} \mathrm{~kg}$. To compensate an annual $\mathrm{CO}_{2}$ emission from the ratio $A E / M_{C 02}^{A}$ we estimate the minimal area at the Earth's surface, $P(A) \%$, to be used for proposed technology and the corresponding details are given at the $6^{\text {th }}$ row of the Table. For our approach the method has to be applied on $2 \%-0.08 \%$ of planet surface, the proposed method can completely compensate the annual carbon emission. To sum up let's estimate the mass of alkali to add for desired modifications in clouds. The mass $\mathrm{KOH}\left(\mathrm{Q}_{\mathrm{KOH}}\right)$ can be estimated using previous data for $n_{K O H}=5.6 \cdot 10^{-3} \mathrm{mg} / \mathrm{l}$ then $p H=10$.Considering the precipitation layer of $1 \mathrm{~m} /$ year for the planet surface $A$ its necessary percent, $P(A)$, can be estimated from the Table. The added mass of alkali at $p H=10$ level is calculated here: $\mathrm{Q}_{K O H}{ }^{10}=n_{K O H} 1 A P(A)=5.7 \cdot 10^{10} \mathrm{~kg}$. Note that similar estimations are presented at the bottom row of the Table 1. In comparison with the fertilizers for soil the added mass of alkali for clouds is relatively small.

Table 1. The carbon mass $\left(M_{C}^{A}\right)$ and $\mathrm{CO}_{2}$ mass $\left(M_{\mathrm{CO}}^{A}\right)$ at the Earth's surface $A=5.1 \cdot 10^{8} \mathrm{~km}^{2}$; the minimal required surface $P(A)$ and mass $Q_{K O H}$ of $\mathrm{KOH}$ to compensate an annual carbon emission.

\begin{tabular}{llll}
\hline 1) $\mathrm{pH}$ & 5.6 & 10 & 11 \\
2) $\mathrm{m}_{\mathrm{C}}{ }^{1}, \mathrm{~kg} / \mathrm{m}^{3}$ & $1.6 \cdot 10^{-4}$ & 0.849 & 26.5 \\
3) $\mathrm{m}_{\mathrm{CO} 2}{ }^{1,} \mathrm{~kg} / \mathrm{m}^{3}$ & $5.9 \cdot 10^{-4}$ & 3.11 & 97.2 \\
4) $\mathrm{M}^{\mathrm{A}}{ }_{\mathrm{C}}, \mathrm{kg}$ & $8.2 \cdot 10^{10}$ & $4.3 \cdot 10^{14}$ & $1.35 \cdot 10^{16}$ \\
5) $\mathrm{M}^{\mathrm{A}}{ }_{\mathrm{CO} 2}, \mathrm{~kg}$ & $3 \cdot 10^{11}$ & $1.6 \cdot 10^{15}$ & $5 \cdot 10^{16}$ \\
6) $\mathrm{P}(\mathrm{A}), \%$ & No possible & $2 \%$ & $0.06 \%$ \\
7) $\mathrm{Q}_{\mathrm{KOH}}$ mil.tons & & 57 & 22.8 \\
\hline
\end{tabular}




\section{The Study for Helicopter Coating and Protection}

The main principles for the laboratory measurements of surface properties for helicopter coating for its improvement are listed below. The purpose is to calculate forces acting on the cloud droplets of different sizes at different surfaces. The water drops were placed at different samples to study and make photographs of the contact area, and then photos were analyzed by computer. The angle $\theta$ and diameter $d_{k}$ of contact of droplets with the surfaces were measured, these parameters are included in the following formula:

$$
\sigma \cdot \cos \theta=\frac{F_{1}}{l}
$$

here $\theta$ is a contact angle of surface and drop of water in contact point, $F_{l}$ is wetting force, $l$ is a wetted perimeter. The surface tension of the water is $\sigma=75.50 \mathrm{mN} / \mathrm{m}$ for temperature $\mathrm{T}=0^{\circ} \mathrm{C}$. The force required to tear the drop from the surface is $F_{2}$, it can be calculated as follows:

$$
\cos \theta \approx \frac{F_{1}}{F_{2}} \quad, \quad F_{2} \approx l \sigma
$$

The surface area of contact $S_{k}$ and perimeter of this area $l$ are calculated as follows:

$$
\mathrm{S}_{\mathrm{k}}=\pi\left(\mathrm{d}_{\mathrm{k}} / 2\right)^{2}, 1=\pi \mathrm{d}_{\mathrm{k}}
$$

Volumes for different drops in experiments were created by medical syringe with high accuracy. The largest drop has volume $U_{5}=0.05 \mathrm{ml}$ with the weight $0.05 \mathrm{~g}$. The smallest drop has the volume of $U_{l}=0.01 \mathrm{ml}$ and $0.01 \mathrm{~g}$ in weight. Also drops were produced and measured for the following volumes: $U_{2}=0.02 \mathrm{ml} ; U_{3}=0.03 \mathrm{ml} ; U_{4}=0.04 \mathrm{ml}$. One can estimate the radius of the drop from known formula for volume as follows:

$$
r=\left(\frac{3 U}{4 \pi}\right)^{1 / 3}
$$

From the formula (7) the free drop radii are $r_{1}=1.337 ; r_{2}=$ $1.684, r_{3}=1.928, r_{4}=2.122, r_{5}=2.229 \mathrm{~mm}$ respectively, and complete surface area $S=4 \pi r^{2}$ of droplet were calculated taking into account the droplet radius. Subtracting the surface area of Sof the droplet and excluding its contact area we obtain the surface area $S_{a}$ that air flow can blow up by the motion:

$$
\mathrm{S}_{\mathrm{a}} \approx 4 \pi \mathrm{r}^{2}-\pi\left(\mathrm{d}_{\mathrm{k}} / 2\right)^{2}
$$

The series of measurements were done as well as calculations using the above procedure. A few typical photographs of water droplets on the surface which were analyzed are shown below in Fig. 3a, b.

The data for duralumin or Teflon surface are shown in Table 2a,b. One can see that large droplets can easily be eliminated from the surface because it takes small value of unified force $F_{2} / S_{a}$ to do it.

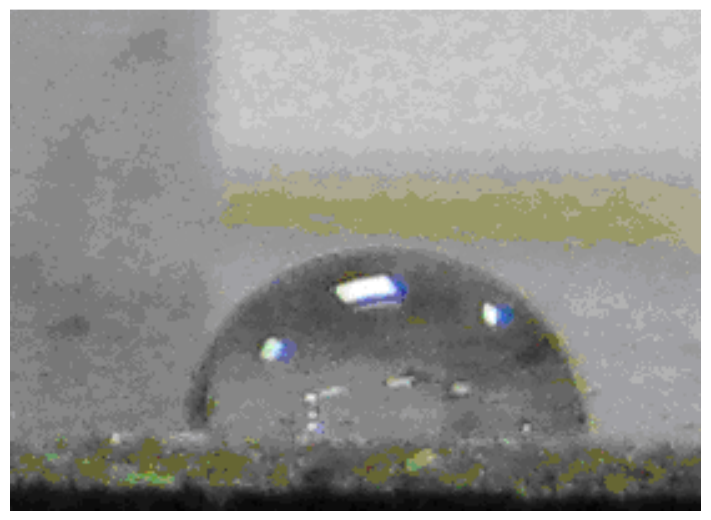

Figure 3a. Water droplets on duralumin surface.

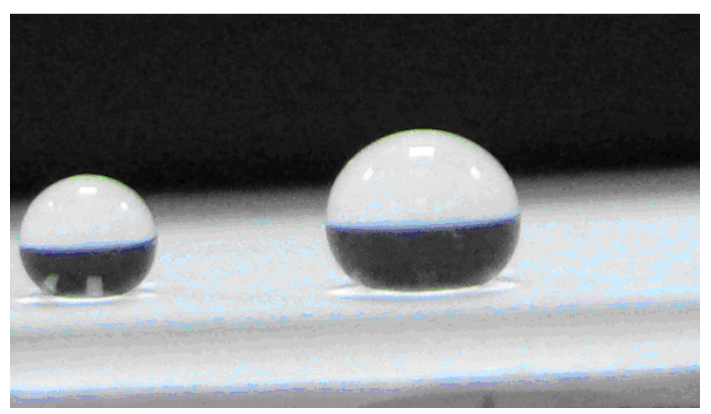

Figure 3b. Droplets on Teflon, their masses are 0.01g; $0.02 \mathrm{~g}$.

Table 2a. Droplet measurements on duralumin surface.

\begin{tabular}{lllll}
\hline $\boldsymbol{M}, \mathbf{G}$ & $\boldsymbol{\theta},^{\circ}$ & $\boldsymbol{d}_{\boldsymbol{k}, \boldsymbol{m} \boldsymbol{m}}$ & $\boldsymbol{F}_{2}, \boldsymbol{m} \boldsymbol{N}$ & $\boldsymbol{F}_{2} / \boldsymbol{S}_{\boldsymbol{a}}, \boldsymbol{N} / \boldsymbol{m}^{\mathbf{2}}$ \\
\hline 0.01 & 60.2 & 3.42 & 0.811 & 61.1 \\
0.05 & 68 & 6.5 & 1.542 & 47.13 \\
\hline
\end{tabular}

Table 2b. Droplet measurements on Teflon surface.

\begin{tabular}{lllll}
\hline $\boldsymbol{M}, \mathbf{G}$ & $\boldsymbol{\theta},^{\circ}$ & $\boldsymbol{d}_{\boldsymbol{k}, \boldsymbol{m} \boldsymbol{m}}$ & $\boldsymbol{F}_{2}, \boldsymbol{m} \boldsymbol{N}$ & $\boldsymbol{F}_{2} / \boldsymbol{S}_{a}, \boldsymbol{N} / \boldsymbol{m}^{2}$ \\
\hline 0.01 & 144.77 & 1.35 & 0.32 & 15.2 \\
0.02 & 146.59 & 2.03 & 0.48 & 14.9 \\
0.03 & 152.21 & 2.48 & 0.588 & 14.0 \\
0.04 & 161.15 & 2.80 & 0.664 & 13.2 \\
\hline
\end{tabular}

Usually, clouds droplets have the radiuses $r_{i}=1-100$ microns, such a small droplets can't be easily obtained in experiments, and further analysis aim to simulate the cloud medium. Let's use the algorithm for laboratory photos as at Fig.3a,b withlarge droplets on the surface to simulate small cloud droplets by approximation, Figure 4. Conversation to very small radii is shown in Fig. 4 by arrow. One can see that the ration is $r / r_{k} \approx 1$ for water/duralumin, so the area of contact of the micro drops is taken here equal to the cross section of the smallest droplets with of $S_{i(r)}=\pi\left(r_{i}\right)^{2}$ due to their good adhesion. The experimental measurement of the quantities $F_{2} / S_{a}$ and $F_{2} / S_{k}$ in Table 2 a was used to calculate the droplet/duralumin dynamics. The force $F_{2}\left(r_{i}\right)$ for smallest droplets in clouds with radius $r_{i}$, measured in meters, can be calculated as follow:

$$
\left(\mathrm{F}_{2} / \mathrm{S}_{\mathrm{k}}\right) \cdot \mathrm{S}_{\mathrm{i}}(\mathrm{r})=88.3 \mathrm{~S}_{\mathrm{i}}(\mathrm{r})=\mathrm{F}_{2}\left(\mathrm{r}_{\mathrm{i}}\right)
$$


For hydrophobic Teflon surfaces, the contact area sure decreases with droplet mass. According to approximation at Fig.4 the small droplets with radius $r$ exhibit much smaller radius in contact $r_{k}$ on the Teflon-type surface, and ratio $r_{k} / r=$ 0.3 of the contact radius to the radius of the drop decreased here. Simulation with the arrow on the experimental plot in Fig.4 indicates the greatest reliability of 0.3 . The breakout force for small cloud droplets can be calculated using the coefficient of proportionality $K$ :

$$
\begin{gathered}
\mathrm{F}_{2}\left(\mathrm{r}_{\mathrm{i}}\right)=\left(\mathrm{F}_{2} / \mathrm{S}_{\mathrm{k}}\right) \cdot \mathrm{K} \cdot \mathrm{S}\left({ }_{\mathrm{i}} \mathrm{r}\right)=22.4 \cdot \mathrm{S}\left({ }_{\mathrm{i}} \mathrm{r}\right) \\
K=\frac{S_{k}\left(r_{i}\right)}{S\left(r_{i}\right)}=\frac{4 \pi r_{i}^{2}}{4 \pi r_{k, i}^{2}}=\left(\frac{r_{i}}{r_{i, k}}\right)^{2} \approx 0.3^{2}=0.1
\end{gathered}
$$

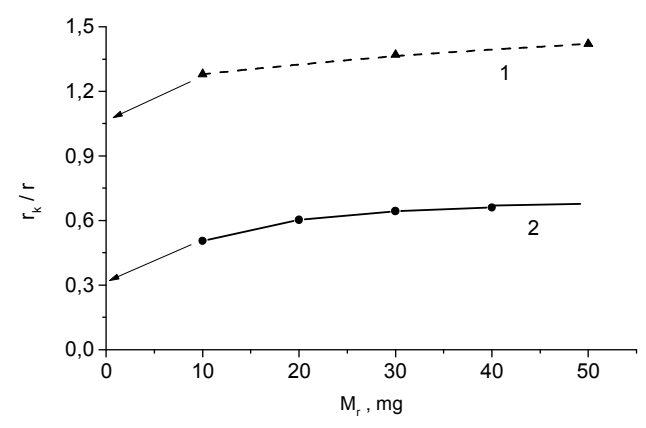

Figure 4. The experimental ratio $r_{k} / r$ of the contact radius as a function of decreasing droplet masses, $M$. The graph 1 displays duralumin surfaces, and the graph 2 is for Teflon.

The results of calculation of forces required for separation of small droplets in typical cloud are shown in Table3.

Table 3. The separation forces $F_{2}$ for droplets with radii $r$.

\begin{tabular}{lll}
\hline$, \boldsymbol{r}, \boldsymbol{\mu m}$ & $\boldsymbol{F}_{2}, \mathbf{N}$, in $(9)$ for duralumin & $\boldsymbol{F}_{2, \mathbf{N}, \text { from }(\mathbf{1 0}) \text { for Teflon }}$ \\
\hline 1 & $2.8 \cdot 10^{-10}$ & $7 \cdot 10^{-11}$ \\
2 & $1.1 \cdot 10^{-9}$ & $2.8 \cdot 10^{-10}$ \\
10 & $2.8 \cdot 10^{-8}$ & $7 \cdot 10^{-9}$ \\
100 & $2.8 \cdot 10^{-6}$ & $7 \cdot 10^{-7}$ \\
\hline
\end{tabular}

Let's analyze different acted forces for tearing droplets from the surface on rotating helicopter blades. Firstly, the centrifugal force $F_{3}$ acts on droplets of different sizes, $r$, on the blades at various distances from the axis of rotation $L_{b}$ and creates the range of droplet speeds, respectively. Typically the velocity $V$ at the end of the blade must not exceed $300 \mathrm{~m} / \mathrm{sec}$, so $V=2 \pi L_{b} f=300 \mathrm{~m} / \mathrm{s}$, with the rotor frequency is $f_{3}=V /\left(2 \pi L_{b}\right)=4.775 \mathrm{~s}^{-1}$, and centrifugal power for the droplet with mass, $M$, used for calculations:

$$
F_{3}=M(r) \omega^{2} L_{b}=\left(4 \pi r^{3} / 3\right) \rho_{w}(2 \pi)^{2} f_{3}{ }^{2} L_{b}
$$

This relation show that the smaller droplets $r=1$ micron cannot be removed by centrifugal force over the entire surface, even at a speed at the end of the helicopter blades $V_{b}$ $=300 \mathrm{~m} / \mathrm{sec}$ for both considered materials, because this value is too small, $F_{3}\left(L_{b}=10\right)=3.76 \cdot 10^{-11} N$, see Table 3 for comparison with $F_{2}$. Droplets of radius $r=2 \mu \mathrm{m}$ can be removed by centrifugal force from rotating blade with Teflon coating only from the 0.7 -meters at the end of its length, because this force is $F_{3}\left(L_{b}=10\right)=3 \cdot 10^{-10} N$. Droplets with radius $r=2 \mu \mathrm{m}$ can not be removed from rotating blade with duralumin surface. Droplets of radius $r=10 \mu \mathrm{m}$ and more ones can be removed by centrifugal force for the length $L_{b}=$ $10 \mathrm{~m}$ from rotating blade with Teflon surface in reality due to 5 times for exceeds of $F_{2}$, so the force $F_{3}\left(L_{b}=10\right)=3.76 \cdot 10^{-8} \mathrm{~N}$. There is partial water removing from duralumin and other no-Teflon coatings according to this algorithm.

In addition, the gravitational force $F_{4}=M(r) g$ acts on a droplets. Calculations show that gravitational force is very small for all considered cloud droplets; this force can exceed the separation force only for big rainy droplets with radius $\mathrm{r}>1 \mathrm{~mm}$.

The force created by flow of air is best for water/surface separation. For large drops on a moving surface one can use the following formula to estimate the drag force:

$$
F_{5}=\frac{\pi r^{2} C \rho_{w}}{2} V^{2}
$$

Taking into account speed the estimate for the Reynolds number here is $R e \sim 50$ (for $V=100 \mathrm{~m} / \mathrm{s}$ and $r=10 \mu \mathrm{m}$ ), so coefficient was set $C=0.4$ in (12). Air drag force in a form (12) can act effectively on the typical cloud droplets with $r>$ $10 \mu \mathrm{m}$ to provide the $F_{5}>F_{2}$. For example then $\mathrm{r}=10 \mu \mathrm{m}$, using equations (12) and (10) one can confirm the last statement for Teflon surface with speed $V>0.3 \mathrm{~m} / \mathrm{s}$. These droplets ensure their separation from duralumin surface at speeds over $0.7 \mathrm{~m} / \mathrm{sec}$ due to equations (12) with (9). In aerosol theory, in particular in the cloud theory of the motion of smallest particles of aerosol, it is assumed that the small droplets are moved by Stokes friction force into the air stream, this force can be determined by the formula:

$$
F_{6}=6 \pi r V \eta_{a}
$$

here $\eta_{\mathrm{a}}$ is a dynamic viscosity of air. This force can tear the small cloud droplets from a moving surface effectively. The smallest droplets can be deleted from the body of the helicopter blades only then $F_{2}>F_{6}$. It means that in practice the smallest droplets are present always at rotating blades of helicopter. For example, from equations (9) and (13) it follows that then $r=5 \mu \mathrm{m}$ the surface speed should be larger then $V=147 \mathrm{~m} / \mathrm{s}$ for duralumin surface. Also this means that half of rotating blades keep such droplets on its surface. On another hand, the Teflon coating was analyzed by comparison of forces in equations (10) and (13), so the speed should be V $=37 \mathrm{~m} / \mathrm{s}$ and this means that more then $10 \%$ percent in a blade length will be free from there droplets.

Developed algorithm and calculations demonstrate that the helicopter body with hydrophobic Teflon-type coating does no hold the most of cloud droplets on its surface. Therefore hydrophobic coating provides a number of benefits and relieves the helicopter no-icing when driving in the clouds with greater conductivity. And additional to it, the smooth 
surface provides reflection of many droplets in comparison with rough one. The additional strong effect is the water vapor condensation and freezing at any moved surface in oversaturated medium at super cooled temperatures. The best way to stop this condensation is to dry of initial wet air as it is considered below.

\section{New Approach for Helicopter to Form Dry Surroundings Around Itself}

A method for reducing aircraft icing involves injecting the calculated amount of absorbent particles (e.g., salts) strictly in the space between the rotating blades [19]. Particles enlarge and get watered by cloud to safe big sizes of droplets. Then particles fall out of the field or screws under gravity or fall to the next blade due to the rotation of the ice-saline droplets. Afterwards particles are removed from the surface by centrifugal force or air stream, since these forces increase with increasing radius drops as $\sim r^{3}, \sim r^{2}$ respectively. These processes deliver the technological result of the complete removal of icing from the blades or/and tail rotor of the helicopter or prevention of its increase after the proposed system in flight. This method provides a significant reduction of energy consumption for antiicing and easy technical implementation.

This technology protects the most important part of aircraft such as the main helicopter rotor providing the moving force. A helicopter is usually located in supersaturated and super-cooled areas of the cloud, so water vapor and droplets deposited on the metal can crystallize very fast. In comparison with airplanes, the greatest difficulty to affect helicopter flight is icing because helicopter flight altitude frequently coincides with the height of the clouds localization Despite recent progress in designing methods for aircraft, this problem still is relevant, but the main advantages of helicopters compared to the plane is their good maneuverability in flight, in many respects due to the ability to hang in certain points of space. At low speeds the movement of the helicopter reduces the impact force of the flow of water drops in the air near the body and screw machine, which at high speeds can lead to failure. Therefore, the aim of the proposed method is to eliminate and prevent the icing of rotating blades of helicopters. To prove the efficiency of the proposed method the general principles of a mechanical device are used in analysis. Other systems related to the known methods and devices of this purpose are described in patents $[20,21,22]$. The most famous and widely used technologies include thermal method employing electric heaters of the front parts of rotating blades located inside them. It is widely used in the current system, but the main disadvantage of all electric systems is high power consumption of on-board power supply of the helicopter [20]. There are known the liquid systems, pneumatic, and other systems. The principle for the application of liquids for mentioned purpose, such as in the patent [23], is icing fluid. In practice for helicopters alcohol is used to act on the windshield of the cockpit. The principal disadvantage of mentioned liquid method is taking large mass of liquid required on board of the aircraft, so in practice it is impossible for the helicopters. The closest to the proposed approach is the method outlined in patent [21]. This method is too complicated; it comprises determining prior using radio measuring and active-passive radar meteorological sensing for vertical and horizontal dimensions recognition of the zones of supercooled clouds. The next step of preparations includes determining the average water content in the radial direction and other characteristics in order to detect the potentially dangerous areas of the cloud. The next step of this method involves an additional airplane fly firstly to do an active influence on these areas of potential helicopter icing by injecting into them the standard reagents that are $\mathrm{AgI}$ or $\mathrm{CO}_{2}$ ice-forming solid reagents to get wet channel in cloud.

It is known that the liquid water content of clouds, $W$, is a basic physical parameter that determines the intensity of icing, $I$, measured in $\mathrm{mm} / \mathrm{min}$ [25]. The mentioned value is proportional to the flight speed $U$, the coefficients of the surface material and its form $\beta_{1}$ and $\beta_{2}$; the icing intensity is $I$ $\sim W U \beta_{1} \beta_{2}$.

According to the proposed method the small calculated amounts of salt having hygroscopic properties should be injected in the area between the rotor blades. The simple core experiment was performed with small helicopter's detail manufactured from duralumin material. The detail was placed in saturated solution of $\mathrm{NaCl}$ to the time period more the one year. No considerable changes at the detailed surface were observed.

The physical mechanism is as follow. Each hygroscopic particle quickly increased in its size absorbing the water vapor around itself directly into the space between the blades of a helicopter rotor. It changes the properties in the falling droplets and air so that the icing of blades no available. Each particle of salt should be watered for the time before next rotating blades that is equal to $t_{h}=1 / f \approx 0.2 \mathrm{sec}$, where $f$ is a frequency of rotation of the rotor. The small salt particle is transformed into a large droplet size, absorbing all the excess moisture from the surrounding volume of air with radius $L_{h p}$. Large drops are safe, they are either fall out from the region under gravitational forces [21], or removed from the blades by centrifugal force or air stream, since these forces increase with increasing droplet radius as $r^{3}$ and $r^{2}$ respectively. These saline droplets fall on the blade that releases salt due to the rotation of the screw of the helicopter and do not cause surface icing of blades. Note that the freezing point of an aqueous salt solution, which the hygroscopic salt materially reduced [26], for $\mathrm{NaCl}-26^{\circ} \mathrm{C}$; for $\mathrm{KCl}-55^{\circ} \mathrm{C}$. In the patent [22] the optimal composition of hygroscopic salts for anti icing agents is given for civiler application that could be used partially here, because magnesium metal and oxides were introduced in this mixture to prevent the corrosion for $\mathrm{Fe}$.

Compared to [20] in the proposed method the main effect of anti-icing is achieved while reducing power consumption in a thousand times, which increases the reliability of piloting of helicopter. Compared to [22], the proposed method is significantly easier to use and require only a small amount of 
reagent $10-30 \mathrm{~kg} / \mathrm{hr}$. Compared to [22], this method provides accurate calculation of the required size and weight of hygroscopic particles related to cloud environments. Usage the proposed method in the industry delivers positive technical and economic effect and significantly increases the reliability of piloting a helicopter in bad weather.

Calculation of basic parameters of the proposed system is shown in example below. It was shown and calculated in [27] that it is sufficient to introduce a small amount, $n$, of hygroscopic salt for a substantial reduction of water content of the treated area. Estimations of watering hygroscopic solid particles such as salts $\mathrm{NaCl}, \mathrm{KCl}$ show that particles from the initial range of $r=10$ microns increased to 22 microns during the time between the release of salt into space and the arrival of the next blade due to rotation at an average water content of the cloud space of $\mathrm{W}=1 \mathrm{~g} / \mathrm{m}^{3}$. As a result droplets of $\mathrm{NaCl}$ will have concentration $\approx 10 \%$ and determine the concentration of solution droplets when the follow-blade propeller rotating at freezing temperature $-13^{\circ} \mathrm{C}$. In the techniques of active influence on the cloud developed now are a large number of hygroscopic particles, which can also be used for purposes of this method. Calculation of the minimal concentration, $n$, and summand mass, $M$, of hygroscopic particles to enter into rotating blade region allows for the diffusion of water supply to the salt particles from its individual volume of radius, $L_{h p}$. This calculation can be carried out by the next formulas:

$$
\begin{gathered}
L_{h p}=\sqrt{D / f} \\
n=\left\{\left(\pi L_{b}^{2} h\right) / L_{h p}^{3}\right\} \\
M=\frac{4}{3} \pi r_{s}^{3} \cdot \rho_{s} \cdot n \cdot K \cdot t_{1}
\end{gathered}
$$

Here, the coefficient of diffusion for small hygroscopic particles and air molecules in a turbulent air was $D=2$ $\mathrm{cm}^{2} / \mathrm{sec}$, while the diffusion time in an individual particle volume between the arrival of the rotating blades $t_{h}=1 / \mathrm{f}=$ $0.21 \mathrm{sec}$. The acted are is $\left(\pi L_{b}^{2} h\right)$, then $L_{b}=10 \mathrm{~m}$ is a length of the blades of a helicopter, and $h=1 \mathrm{~m}$ is a thickness entrained air vane. The $\mathrm{K}(=4)$ is number of blades. The $\rho_{\mathrm{s}}=$ $2 \mathrm{~g} / \mathrm{cm}^{3}$ is a density of salts, so $L_{h p}=6.5 \mathrm{~mm}$ is a characteristic diameter of the individual volume of each hygroscopic particle. The $t_{l}=1 h$ is a time fly unit of one hour. In the example we get the estimated mass of salt $M=$ $33 \mathrm{~kg} / \mathrm{h}$ that can be easy placed into helicopter in advance. The required mass may be reduced as a result of experiments taking into account long time of freezing brine.

Structurally, the system of delivering the air/salt mixture can be organized on the principle of dynamic sirens [3]. The stator is a stationary part with openings of the holes through which compressed air is periodically goes from central stator into the duct blade. The stator holes located outside of the rotating rotor holes that provide periodic coincidence holes, so compressed air can fall to the resonator with strong sound. The scope of the stator can have here a low power pump about 100
$\mathrm{W}$ and it is injected an air/salt mixture under pressure exceeding with 3 and to 5 atmospheres.This procedure provides the directional movement of the air. The resulting mass of hygroscopic particles emitted can easily be adjusted directly in the air in a controlled manner by varying the pressure in the volume of air/salt mixture during fly. The one implementation of the method to put particles is presented at Fig.5, where salty particles go away from small holes to opposite side in front of each blade. Another implementation is a common hole from additional tube located at the upper rotating blades to put particles to common area in particular in the back half of this spherical area.

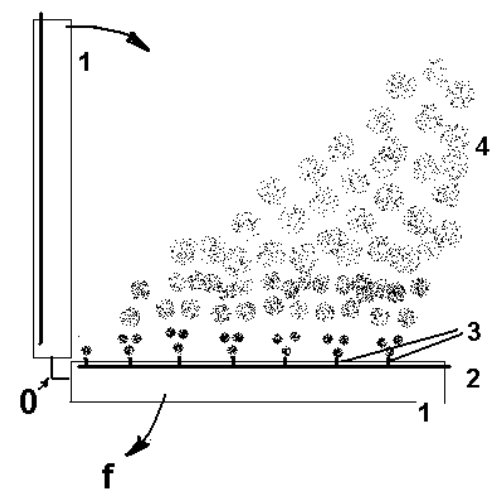

Figure 5. Schematic representation of the method for removing ice from a helicopter rotor;

1 - rotating the rotor blade at a frequency $\mathrm{f}, 0$ is the place of rotation of the blades;

2 - duct for supplying the hygroscopic particles;

3 - holes in the duct;

4 - hygroscopic salt particles, flooded in the cloud space between the blades.

\section{Conclusion}

Helicopter is a very convenient instrument for precipitation enhancement, and additional proposed methods for reducing icing provide the freely fly inside natural clouds.

\section{Acknowledgements}

The authors are very grateful to Professors Thomas W. Choularton, Peter M. Cox, Yury A. Israel, Feodor V. Bunkin, Alexander Nadezhdinskii and Jean Charles Munch for useful consideration and to Dr. Svetlana Amirova for the help in a work.

\section{References}

[1] IPCC: Summary for Policymakers, In: "Climate Change 2014, Mitigation of Climate Change. Contribution of Working Group III to the Fifth Assessment Report of the Intergovernmental Panel on Climate Change", Edenhofer, O., R. Pichs-Madruga, Y. Sokona, E. Farahani, S. Kadner, K. Seyboth, A. Adler, I. Baum, S. Brunner, P. Eickemeier, B. Kriemann, J. Savolainen, S. Schlömer, C. von Stechow, T. Zwickel and J.C. Minx (eds). Cambridge University Press, Cambridge, United Kingdom and New York, NY, USA, 2014. 
[2] R.T. Bruintjes. "A review of cloud seeding experiments to enhance precipitation and some new prospects". BAMS, v.80, 1999, pp.805-820.

[3] T. Tulaikova, S. Amirova. "The effective possibility for atmosphere $\mathrm{CO}_{2}$ purification", 2012, Lap-Lambert, Germany

[4] Svetlana Amirova, Tamara Tulaikova. "One possibility for atmosphere $\mathrm{CO}_{2}$ purification to get climate recovery". Science Discovery in Science PG. Special Issue: New Technical Ideas for Climate Recovery. Vol. 3, No. 1-2, 2015, pp.1-6.

[5] T. Tulaikova, S. Amirova. "The method for effective $\mathrm{CO}_{2}$ purification in the atmosphere". Global Journal of Science Frontier Research. Vol. 15-H, No.1, Version 1.0, 2015, pp. 1-9.

[6] I. Langmuir, I. and V. Schaefer. "Improved methods of conditioning surfaces for adsorption" J. Am. Chem. Soc., 59, 1937, pp.1762-1763.

[7] A.S. Drofa. et al. "Formation of cloud microstructure: the role of hygroscopic particles". Izvestiya. Atmospheric and oceanic physics, v.42, 2006, pp. 355-366.

[8] S.M Shmeter, G.P. Beryulev. "Efficiency of cloud and precipitation modification with hygroscopic aerosols". Rus. Meteorology and Hydrology, v.2, 2005, pp. 43-60.

[9] Mednikov, A.P. "Acoustic coagulation and precipitation of aerosols". New York, Consultants Bureau, 1965.

[10] T.V Tulaikova, A.V. Michtchenko, S.R. Amirova. "Acoustic rains". Moscow, Physmathkniga-press, 2010

[11] H. Yunge. "Chemical compounds and radio-activity in atmosphere". Clarendon, Oxford, 1965.

[12] S.I Rasool, ed. "Chemistry of the lower atmosphere". Plenum, New York, 1973.

[13] E.K. Bjutner. "The influence of pollution of the ocean surface to transport for heat, gas and humidity". Hydromet-press, Leningrad, 1985.

[14] Edwin A. Roehl JR., Ruby C. Daamen, John B. Cook. "Estimating seawater intrusion impacts on coastal intakes as a result of climate change". Journal - American Water Works Association,. http://dx.doi.org/10.5942/jawwa2013.105.0131
[15] H.C. Broecher, J. Peterman. "The influence of wind on $\mathrm{CO}_{2}$ exchange in a wind-water tunnel including the effect of minelayers". J. Mar. Res., V. 36, 1978, pp. 595-610.

[16] R. Wanninkhof, M. Knox. "Chemical enhancement of $\mathrm{CO}_{2}$ exchange in natural water". Limnol. Oceanogr., v. 41, 1996, pp. 689-68.

[17] S. Kobayashi, T. Oguchi, S. Tanelli, E. Im. "Backscattering enhancement on spheroid-shaped hydrometeors: Considerations in water and ice particles uniform size and Marshall-Palmer distributed rains". Radio Science, V. 42, doi: 10.1029/2006RS003503.

[18] "The Pike. Method for determination of wet solder materials". GOST 23904-79, from 11.29.1979, Moscow, Russia.

[19] T.V. Tulaikova. "A method for the elimination and prevention of icing of the rotor of the helicopter in the clouds". Patent Ru2541548 from 24.07.2013.

[20] G.G. Blutshtein, E.V. Busigin, S.G. Golberg, V.V. Nam. "Electro heating anti-icing system, for helicopters for example”. Patern RU 2226481 from 19.07.2002.

[21] V.D. Stepanenko, G.G. Shukin at ell. "The method for decrease the icing for aircraft". PatentRU 2228883, from 11.01.2002.

[22] Yu. S. Saphrigin, Yu. V. Buksha at ell. "The method of anti icing substrat”. Patent RU 2212428, from 25.04.2002.

[23] R Heikky, Yu.-P. Nieminen. "The anti icing liquid for aircraft and related roads”. Patent RU 2191199, from 26.08.1998.

[24] A.A. Chernikov, G.P. Beriulev, V.P. Korneev. "The method of active action for clouds". Patent RU 2295231 from 07.07.2005.

[25] I.P. Mazin. "Physical fundamentals of aircraft icing". Moscow: Gidrometeoizdat, 1957.

[26] E.V. Zielinska, E.Y. Voronin. "Theoretical aspects of the use of hydro resources". Moscow: The Academy of Natural Sciences, 2009 .

[27] Y. S. Sedunov. "Physics of liquid-phase in the atmosphere". Leningrad: Gidrometeoizdat, 1972. 